\title{
Schizophrenics' difficulties in understanding ambiguous sentences
}

\author{
PEGGY A. ROTHBAUM \\ University of North Carolina at Chapel Hill, Chapel Hill, North Carolina 27514 \\ DONALD K. ROUTH \\ University of Iowa, Iowa City, Iowa 52242 \\ and \\ D. LYNNE FEAGANS, LUCRETIA KINNEY, and ELLEN VASU \\ University of North Carolina at Chapel Hill, Chapel Hill, North Carolina 27514
}

\begin{abstract}
Schizophrenic and control subjects were presented with sentences containing lexical, surfacestructure, or underlying-structure ambiguity or no ambiguity and were asked to choose pictures representing the meanings of the sentences. Schizophrenics were significantly impaired in detecting all three types of ambiguity and, in addition, displayed a bias toward the primary meanings of sentences. They were also significantly impaired relative to controls in dealing with unambiguous sentences. However, this was found to be due to excessive response to distractor pictures rather than to lack of understanding of the unambiguous sentences.
\end{abstract}

This study explored whether schizophrenics have difficulty in dealing with linguistic ambiguity. Investigation of how schizophrenics (and others) resolve such ambiguity in sentences is an important route to knowledge about their language comprehension in general. This is true because, as Cairns (1973) points out, many of the ordinary (ultimately unambiguous) sentences we hear are ambiguous at some point prior to their conclusions.

Several levels of linguistic ambiguity have been identified (Chomsky, 1965). One level, lexical ambiguity, is illustrated by the sentence, "The man is scrubbing the big trunk," in which the word "trunk" could refer to either an elephant's trunk or a steamer trunk. A second level, surface-structure ambiguity, has to do with how words are grouped into phrases within the sentence. Thus, in the sentence, "Small boys and girls are frightened easily" (MacKay \& Bever, 1967), it is not clear whether the word "small" applied to both boys and girls or only to boys. A third level, underlying-structure ambiguity, cannot be clarified by regrouping words or by changing intonation. For example, in the sentence, "The mayor will ask the police

A senior honors thesis at the University of North Carolina carried out by the first author and directed by Alice Gordon served as pilot work for the present study. The assistance of W. J. Eichman, Jr., J. L. Lubker, S. McHale, and P. W. Meilman is gratefully acknowledged. The research was supported in part by U.S. Public Health Service, Maternal and Child Health Project 916, and by Grants HD-03110 and ES-01104 from the National Institutes of Health. Requests for reprints should be sent to Donald K. Routh, Department of Psychology, University of Iowa, Iowa City, Iowa 52242. to stop drinking," it is unclear whether the police are themselves to cease drinking or to restrain others from drinking.

MacKay (1966) asked undergraduates to complete sentence fragments containing lexical, surface-structure, or underlying-structure ambiguity and found that completion time varied directly with the level of ambiguity involved. Schulz and Pilon (1973) had school children listen to tape-recorded ambiguous sentences, paraphrase them, and respond to pictures illustrating the two possible meanings of each sentence. They found that the ability to detect lexical ambiguity develops at an earlier age than detection of surface- and underlyingstructure ambiguity, which did not emerge until age 12 .

Of the three levels of ambiguity, research with schizophrenic subjects has so far dealt mainly with the lexical type. Chapman and Chapman (1965) hypothesized that schizophrenics rely excessively on the "strong" meanings of words (the meaning most likely to occur to one when the word is encountered in isolation), with the "weak" meanings having little influence. Since ambiguity, at least at the lexical level, has to do with both the strong and weak meanings of words, the implication is clear that schizophrenics should have difficulty in detecting this type of linguistic ambiguity. Benjamin and Watt (1969) found, as the Chapman and Chapman hypothesis predicted, that schizophrenics were more likely than controls to select the primary meanings for ambiguous words in sentences. This tendency was not attributable to any lack of knowledge of the secondary meanings of the words. Blaney (1974) stated the possibility of extending the Chapman and Chapman hypothesis to syntactic as well 
as lexical ambiguities, but the actual sentences employed in Blaney's experiments were not ambiguous.

The present study assessed the ability of schizophrenics to detect linguistic ambiguity at each of the three levels described above. Partly on the basis of the Chapman and Chapman (1965) hypothesis, it was expected that schizophrenics would have trouble with lexical ambiguity, and perhaps with surface- and underlying-structure ambiguity as well. It was thought that when they chose only one meaning of a sentence, they would be more likely than controls to choose the stronger or more preferred meaning of the sentence.

\section{METHOD}

\section{Subjects}

The 20 schizophrenic subjects were chosen from patients on the admissions ward of John Umstead Hospital, Butner, North Carolina. In an attempt to minimize drug effects on the subjects' responses, all were tested within 3 months, and all but one within 6 weeks, of admission. All had a hospital diagnosis of schizophrenia and were judged by ward physicians to be sufficiently alert and cooperative to leave the ward to go to the experimenter's office for participation in the study. In order to confirm the diagnosis of schizophrenia for research purposes, patients were interviewed in standard fashion with items from the Mental Status Schedule (Spitzer, Fleiss, Burdock, \& Hardesty, 1964). The patients selected for the study were rated independently by the interviewer and an observer as showing at least three out of the following four symptoms: loose associations, delusions, loss of ego boundaries, and autism. Newmark, Raft, Toomey, Hunter, and Mazzaglia (1975) found that, using these four symptoms, they could correctly classify $97 \%$ of psychiatric in-patients as schizophrenic or nonschizophrenic. In the present research, both raters had to agree perfectly on the presence or absence of each of the four symptoms for a patient to be accepted for study.

The schizophrenic sample consisted of 12 males and 8 females. It was not administratively possible to obtain demographic information from patients' files, but summary statistics on the entire hospital indicate a mean patient educational level of Grade 10 and a typical lower socioeconomic class background. It might be presumed that these acute, alert, and cooperative schizophrenics were somewhat better educated than the hospital average. Their age range was 18 to 55 years.

The 20 control subjects were firemen employed by Chapel Hill, North Carolina. Again, it was not administratively possible to obtain detailed demographic information, but according to official job requirements, the firemen would generally have completed Grade 12 . Their age range was 20 to 45 years.

An additional group of 20 paid college student volunteers ( 10 male and 10 female) were recruited by advertisements on the University of North Carolina campus. These subjects provided the data needed to determine the primary or preferred meanings of the sentences used.

\section{Stimulus Materials}

In all, there were 4 practice sentences and 30 experimental sentences, each sentence being presented along with a set of four line drawings in a manilla folder. Two of the practice sentences were ambiguous, and two were unambiguous. Of the 30 experimental sentences, 12 were unambiguous, 6 contained a lexical ambiguity, 6 a surface-structure ambiguity, and 6 an underlyingstructure ambiguity. For the unambiguous sentences, one of the four corresponding pictures correctly represented the meaning of the sentence, and the other three pictures were incorrect distractors. For the ambiguous sentences, two of the pictures correctly represented the two alternative meanings, and the other two pictures were distractors. For example, one sentence with a surface-structure ambiguity was "The girl is bringing the cowboy hats." One of the correct pictures showed a girl bringing policeman hats to a cowboy, and the other showed a girl bringing cowboy hats to a policeman. The sentences were the same ones used by Gordon and Walton (Note 1), who found that with this procedure, children as young as 7 years performed as well as those aged 16 years in the Schulz and Pilon (1973) study.

\section{Procedure}

The experimenter tested the schizophrenic and control subjects individually. She began by reading each of the practice sentences and showing the corresponding sets of pictures. After it was clear that the subject understood the concept of ambiguity and knew how to respond, the actual experiment began. From this point on, the sentences were played from a tape, and the subject was asked after each sentence to choose the picture or pictures that went with that sentence. After the subject made one choice from a set of pictures, the experimenter always asked if there were any others, to insure that all pictures were considered. The sentences were presented in a single fixed sequence, in which the control sentences and those with different types of ambiguity were well mixed.

The paid student volunteers were played the tapes of the same sentences and shown the pictures in small groups rather than individually. They were not required to detect ambiguity but were asked to choose for each item the one picture that best illustrated the meaning of the sentence conveyed. The preferred meaning was defined as the one chosen by a majority of these subjects. The alternate meaning was thus considered to be the nonpreferred meaning of the sentence.

\section{RESULTS}

Error-free performance on a sentence consisted of choosing the one or two correct alternatives pictured, plus avoidance of all distractor pictures. Table 1 presents the means and standard deviations for the number of each type of sentence performed in error-free fashion by schizophrenics and control subjects. The schizophrenics correctly detected $52 \%$ of the lexical ambiguities, whereas control subjects correctly detected $90 \%$ of such ambiguities. Of the surface-structure ambiguities presented, schizophrenics correctly detected $20 \%$, and control subjects detected $48 \%$. Of the underlyingstructure ambiguities presented, schizophrenics correctly detected $28 \%$, and control subjects $65 \%$. There were also differences in correct performance on the unambiguous sentences presented: Schizophrenics were error free on $66 \%$ of them, while controls were error free on $86 \%$.

Table 1

Number of Sentences Performed in Error-Free Fashion

\begin{tabular}{|c|c|c|c|c|c|}
\hline \multirow{2}{*}{$\begin{array}{c}\text { Type of Ambiguous } \\
\text { Sentence }\end{array}$} & \multirow[b]{2}{*}{$\mathbf{N}^{*}$} & \multicolumn{2}{|c|}{ Schizophrenics } & \multicolumn{2}{|c|}{ Controls } \\
\hline & & Mean & SD & Mean & SD \\
\hline Lexical & 6 & 3.1 & 2.2 & 5.4 & 1.0 \\
\hline Surface Structure & 6 & 1.2 & 1.3 & 2.9 & 1.6 \\
\hline Underlying Structure & 6 & 1.7 & 1.4 & 3.9 & 1.9 \\
\hline Unambiguous & 12 & 8.0 & 2.3 & 10.4 & 1.5 \\
\hline
\end{tabular}

*Number of sentences presented. 
Preliminary statistical analysis of the schizophrenic sample above showed no significant sex difference on this (or any other) variable. Thus, male and female schizophrenics were combined for subsequent analysis. A multivariate analysis of variance on the data for sentences performed in error-free fashion showed a significant overall difference between schizophrenic and control samples $[F(4,35)=14.210, p<.001]$. The differences between the groups were also significant for each type of sentence presented [i.e., lexically ambiguous sentences, $\mathrm{F}(1,38)=17.853, \mathrm{p}<.001$; surfacestructure ambiguous sentences, $F(1,38)=12.353$, $\mathrm{p}<.001$; underlying-structure ambiguous sentences, $\mathrm{F}(1,38)=18.031, \mathrm{p}<.001 ;$ and unambiguous sentences, $\mathrm{F}(1,38)=15.043, \mathrm{p}<.001]$.

Although it is somewhat redundant to do so, one may also analyze two separate components of the error-free sentences measure, namely, the number of correct choices made and the number of distractors chosen. For all types of ambiguous sentences, there were significant differences between schizophrenics and control subjects on both of these component measures; that is, schizophrenics made significantly fewer correct choices and chose significantly more distractors. Thus, in the case of ambiguous sentences, this analysis added little to the analysis already presented.

For unambiguous sentences, however, this subsidiary analysis was more revealing. On these 12 sentences, schizophrenics chose the correct picture on a mean of $10.7(\mathrm{SD}=1.5)$, while control subjects chose the correct picture on a mean of $11.4(\mathrm{SD}=.9)$. The difference between groups was nonsignificant. However, on the same 12 unambiguous sentences, schizophrenics chose a mean of 4.4 distractor pictures $(\mathrm{SD}=3.2)$, while control subjects chose a mean of 1.7 distractors $(S D=1.6)$. In this case, the difference between groups was significant $[F(1,38)=11.713, p<.001]$.

Of the 18 ambiguous sentences, it was possible to define a primary or preferred meaning for 16 (for the other 2 sentences, half of the college students chose each meaning). Schizophrenics chose only the preferred meaning of .34 of the ambiguous sentences ( $\mathrm{SD}=.16$ ), while control subjects chose only the preferred meaning of .23 of the sentences $(S D=.20)$, a significant difference $[F(1,38)=21.12, p<.001]$. To examine the differential responses of the schizophrenic and control subjects to the two meanings of these sentences in another way, a standard (z) score was calculated for each sentence. When only one meaning was chosen, schizophrenics chose the preferred meaning significantly more often than the nonpreferred meaning in 12 out of the 16 sentences, while the control subjects (firemen) did so on only 4 out of the 16 sentences.

\section{DISCUSSION}

The results of this study in general confirmed the hypothesis on which the study was based. Schizophrenics were less able than control subjects to detect not only lexical ambiguity, but also ambiguity in the surface or underlying structures of sentences. The data were consistent with the Chapman and Chapman (1965) hypothesis about schizophrenic bias toward the "strong" or primary meanings of words and, in addition, supported Blaney's (1974) suggestion that they have a similar bias toward the primary meanings of sentences as well. If it is true, as Cairns (1973) suggests, that understanding ordinary sentences involves resolving ambiguities of the type studied here, the results may point to a major source of schizophrenics' well known communication difficulty.

One unanticipated finding was that schizophrenics had difficulty even with the unambiguous sentences presented them. Subsequent analysis of the data for these sentences suggested, however, that the source of the difficulty was not in understanding the sentences as such (there was no significant difference between schizophrenics and controls in number of correct alternatives chosen). Rather, the schizophrenics' difficulty with these sentences seemed to be due to their undue responsiveness to the distractor pictures. This finding does not seem surprising in relation to the research literature. Chapman (1956) long ago noted that schizophrenics were much more negatively affected than control subjects by visual distractors, and subsequent studies using a variety of methods have confirmed the hypothesis of excessive schizophrenic distractibility (e.g., Chapman, 1958; Deckner \& Blanton, 1969; Downing, Shubrooks, \& Ebert, 1966).

There are a number of limitations of the present study that suggest that it be considered a preliminary exploration of its topic. Ideally, such research needs to include drug-free schizophrenic patients and groups representing the full range of chronicity. Blaney (1974), for example, in his study of schizophrenic language, found different results with groups varying in degree of psychotic disorganization. It would also have been preferable to have better demographic matching of patient and control groups, and to have tasks matched on reliability, mean, variance, and shape of distribution (Chapman \& Chapman, 1973). Nevertheless, it is the first research known to the authors demonstrating schizophrenic deficits in the detection of surfacestructure and underlying-structure and well as lexical ambiguity.

\section{REFERENCE NOTE}

1. Gordon, A. M., \& Walton, M. D. Children's detection of linguistic ambiguity. Unpublished manuscript, University of North Carolina, Chapel Hill, 1975.

\section{REFERENCES}

Benjamin, T. B., \& Watt, N. F. Psychopathology and semantic interpretation of ambiguous words. Journal of Abnormal Psychology, 1969, 74, 706-714.

Blaney, P. H. Two studies on the language behavior of schizophrenics. Journal of Abnormal Psychology, 1974, 83, 23-31.

CaIRns, H. S. Effect of bias on processing and reprocessing of lexically ambiguous sentences. Journal of Experimental Psychology, 1973, 97, 337-343.

Chapman, L. J. Distractibility in the conceptual performance of schizophrenics. Journal of Abnormal and Social Psychology, 1956, 53, 286-291.

Chapman, L. J. Intrusion of associative responses into schizophrenic conceptual performance. Journal of Abnormal and Social Psychology, 1958, 56, 374-379.

Chapman, L. J., \& ChapMan, J. P. Interpretation of words in schizophrenia. Journal of Personality and Social Psychology, 1965, 1, 135-146.

Chapman, L. J., \& Chapman, J. P. Problems in the measurement of cognitive deficit. Psychological Bulletin, 1973, 79, 380-385. 
Сномsкy, N. Aspects of the theory of syntax. Cambridge, Mass: MIT Press, 1965.

DECKNER, C. W., \& Blanton, R. L. Effect of context and strength of association on schizophrenic verbal behavior. Journal of Abnormal Psychology, 1969, 74, 348-351.

Downing, R. W., Shubrooks, S. J., \& Ebert, J. N. Intrusion of associative distractors into conceptual performance by acute schizophrenics: Role of associative strength. Perceptual and Motor Skills, 1966, 22, 460-462.

MacKay, D. G. To end ambiguous sentences. Perception \& Psychophysics, 1966, 1, 426-436.

MacKay, D. G., \& Bever, T. G. In search of ambiguity. Perception \& Psychophysics, 1967, 2, 193-200.
Newmark, C. S., Raft, D., Toomey, T., Hunter, W., \& Mazzaglia, J. Diagnosis of schizophrenia: Pathognomonic signs or symptom clusters. Comprehensive Psychiatry, 1975, 16, 155-163.

Schulz, T. R., \& Pilon, R. Development of the ability to detect linguistic ambiguity. Child Development, 1973, 44, 728-733.

Spitzer, R. L., Fleiss, J. L., Burdock, E. I., \& Hardesty, A. S. The Mental Status Schedule: Rationale, reliability, and validity. Comprehensive Psychiatry, 1964, 5, 384-394.

(Received for publication January 3, 1979.) 